\title{
Throughput Performance of Transport-Layer Protocols over Wireless LANs
}

\author{
Antonio DeSimone \\ tds@hoserve.att.com
}

\author{
Mooi Choo Chuah \\ chuah@hoserve.att.com
}

\author{
On-Ching Yue* \\ ocyueQie.cuhk.hk
}

AT\&T Bell Laboratories, Holmdel, NJ 07733-3030 USA

\begin{abstract}
We consider the performance of transport-layer protocols over networks where one of the links is wireless. A reliable transport protocol is reponsible for end-to-end data integrity, and will retransmit lost or errored data. Compared to a link over copper or fiber, a wireless link will have a much higher error rate. Link-layer retransmissions can reduce the error rate on the radio link, but the interaction of link-layer retransmission with end-toend retransmission can be complicated. We have investigated, via analytic, numerical and simulation techniques, the end-to-end effects of link-layer retransmissions in the presence of a reliable end-to-end transport protocol. Our results identify the mechanisms affecting end-to-end performance when retransmissions are used to get better error performance on a link. We quantify the increased load on the link due to competing retransmission strategies, and, for a transport protocol modeled on TCP, we identify the region of loss rates where link-layer retransmissions have the undesirable effects of both reducing end-to-end throughput and increasing link utilization in the network segment where bandwidth is the most expensive.
\end{abstract}

\section{Introduction}

Advances in digital signal processing, wireless transmission and wireless multiple access techniques, together with the proliferation of cellular telephones and wireless data products, have generated growing interest among telecommunications researchers in Personal Communication Systems (PCS)[1][2]. While most of the research activities in PCS have been in the area of voice communications, there is also an emerging interest in providing wireless data services. There are already products on the market to provide a wireless link between the end user's computer and a network access point, such as a gateway, to complete the connection to the remote host via a wide area network. From a networking point of view, replacing the last link with a wireless one introduces several

\footnotetext{
*currently on leave at the Department of Information Engineering at the Chinese University of Hong Kong
}

design considerations because wireless link may be less secure and less reliable. In this paper we shall focus on the unreliability of the wireless link, and on the tradeoffs in alternate error recovery strategies to ensure data integrity over a connection that includes a wireless link.

While there are other physical transmission medium for wireless communication links, such as infrared, the most likely choice is radio. [3] Several factors contribute to the unreliability of radio links in the network. First, the radio frequency spectrum is a very valuable resource and the bandwidth allocated to PCS will always be limited. Second, the propagation environment causes reverberations, resulting in a large dynamic range in the received power. Finally, the transmitted power is limited because of interference and battery conservation considerations. Consequently, the bit error rate (BER) on radio link is typically higher than that of wired connections. The critical issues in data networking over wireless links is the transport-layer protocol performance in the presence of noisy links.

A transport protocol is responsible for end-to-end data integrity. A reliable, connection-oriented transport protocol will provide correct, in-sequence communications even over unreliable networks by detecting lost and duplicate packets and requesting retransmission of lost packets. The data throughput performance of the transport protocol is ultimately what is seen by the application. In turn, the performance of the transport protocol depends on the service provided by the underlying network and link layers. The network is assumed to provide an unreliable service, in the manner of IP, CLNP or frame relay networks, and that data integrity and reliability is the responsibility of the end-to-end transport protocol. The link layer, and the wireless link in particular, may provide either unreliable, best-effort service or reliable delivery via retransmissions.

One mechanism used by transport protocols to detect packet loss is based on acknowledgments and timers. All transmitted data is acknowledged by the receiver, and a lost packet is signaled by the expiration of a timer at the transmitter before the acknowledgment arrives. We call 
this end-to-end error recovery mechanism transport-layer retransmissions (TLR). TCP[4] and TP4 are examples of transport protocols that operate in this manner.

The performance of TLR depends critically on the value of the retransmission timeout, $\tau_{R T O}$. The $\tau_{R T O}$ must be small enough to respond quickly to loss, but not so small as to allow the retransmission of data that is only delayed in the network and not lost. A key input to the $\tau_{R T O}$ is the measured round-trip time, $\tau_{R T T}$, between the source and destination: clearly, we would like to have $\tau_{R T O}>\tau_{R T T}$ to avoid unnecessary retransmissions. $\tau_{R T T}$ estimates are obtained from measurements of the delay of acknowledgments at the transmitter.

A possible solution to the high BER on a wireless link is to add link-layer retransmission (LLR) to reduce the link error rate. For the transport protocol, the reduction in link error rate from LLR comes at a cost. LLR increases the round-trip times and the variability in roundtrip times seen by a transmitter. Retransmissions at the link layer affect $\tau_{R T T}$ estimation and therefore can affect the performance of a transport protocol in non-trivial ways.

\section{Related Work}

Edge[5] compared two approaches to network interconnection: the end-to-end approach (which we refer to as TLR) and the hop-by-hop approach (similar to the LLR approach). The performance metrics of interest were endto-end delay and storage requirements at the network nodes. Biswas et al.[6] investigated the performance of a multiple access protocol for an ATM-based pico-cellular radio LAN. The retransmission protocol is a simple timeout strategy, with a fixed $\tau_{R T O}$ and back-off. Bae et al.[7] investigated both the TLR and LLR schemes in ATM environments. Their paper considers TLR and LLR as alternatives, but does not look at the interaction of the two schemes. Unlike the ATM environment, where the bit error rate is assumed to be small, we are interested in the high packet loss rates characteristic of the wireless environments. The analysis in [7] proceeds by making the standard independence assumptions and assuming that the transmission delays are exponentially distributed. As in [5] and [6], the model assumes a fixed $\tau_{R T O}$.

\section{Road Map}

This paper looks at simple models of the performance of transport protocols, using analytic, numerical and simulation techniques. The analysis allows a comparison of end-to-end performance with and without link-layer retransmission, to evaluate the tradeoffs. First we study how LLRs affect on round-trip time estimation. LLRs change the transport layer behavior by increasing the $\tau_{R T T}$ values seen at the transmitter. Section 4 presents some analytic results on the sensitivity of end-to-end throughput to variability in delay, for the case of fixed $\tau_{R T O}$. Section 5 looks specifically at the effect of variability in delay due to LLR on TCP's adaptive calculation of $\tau_{R T O}$, both numerically and by simulation. Second, the transport protocol may retransmit data that is delayed, but ultimately delivered successfully by LLR. The duplicated data will be discarded by the receiver, ensuring reliable data transfer, but transmission bandwidth on the link employing LLR will have been wasted. ${ }^{1}$ This effect is also discussed in Section 5. Note that, unlike the $\tau_{R T O}$ effect, the unnecessary retransmissions affect all users of the link, not just the one transmitter and receiver. Therefore, in addition to throughput, we are interested performance measures that indicate wasted capacity on the wireless link, and in Section 5 we look at the number of link transmissions required to successfully deliver a packet to the application.

\section{Analytical Results for Static RTO}

In this section, we investigate how the throughput is related to the packet loss probability and the distribution of the round trip delay. We have taken an analytical approach in order to gain insight into the relationships among the various system parameters. However, to keep the analysis from being intractable, we limit ourselves to the case of fixed $\tau_{\text {RTO }}$ settings.

For the static $\tau_{R T O}$ case, the throughput of the version of TCP under study in this paper-one-at-a-time selective repeat [8] - is bounded by the throughput of the go-back- $\mathrm{N}$ flow control protocol. ${ }^{2}$ Therefore the starting point of our analysis is the familiar formula for the throughput of go-back-N[9]:

$$
T=C \frac{1-p}{1+p\left(C \tau_{R T O}-1\right)}
$$

where $\mathrm{C}$ is the link capacity, $p$ is the probability of retransmission and $\tau_{R T O}$ is the retransmission timeout setting. We also make the usual assumptions about statistical independence between packet loss and delay.

We classify the two causes of retransmission due to RTO expiration into two types: due to packet loss and due to delay. Denoting the probabilities of the two types of retransmissions as $p_{l}$ and $p_{d}$, we have

$$
p=p_{l}+\left(1-p_{l}\right) p_{d}
$$

${ }^{1}$ This points out the importance of looking at end-to-end performance. At the link layer, both packets count as correct transmissions, but to the transport protocol, only one contributes to useful throughput.

${ }^{2} \mathrm{TCP}$ as usually implemented is not go-back-N, but since only one segment can be recovered in as round-trip time, the per-connection throughput is no better. 
While $p_{l}$ is related to the BER of the physical link, ${ }^{3} p_{d}$ is the probability that the actual $\tau_{R T T}$ is larger than $\tau_{R T O}$. We refer to transmissions due to excess delay as spurious retransmissions. $p_{d}$ is a function of $\tau_{R T O}$, and can be computed from the probability distribution function of a random variable which corresponds to the difference between the round trip delay and the transmission time of one packet at link capacity, $\operatorname{P(t)}=\operatorname{Pr}\left(\tau_{R T T}-\frac{1}{C}<t\right)$ :

$$
\mu_{d}\left(\tau_{R T O}\right)=\int_{\tau_{R T O}-\frac{1}{C}}^{\infty} d P(t)
$$

We shall take the mean of the random delay as $m$ and denote the variance as $\sigma_{d}^{2}$. Equation 1 is rewritten as follows with $C=1$ to show the dependence of throughput on the setting of $\tau_{\text {FTO }}$ explicitly:

$$
T\left(\tau_{R T O}\right)=\frac{1-p\left(\tau_{R T O}\right)}{1+\left(\tau_{R T O}-1\right) p\left(\tau_{R T O}\right)}
$$

If the round trip delay is deterministic, that is, $\sigma_{d}^{2}=0$, we can pick $\tau_{F T O}>m$ and the RTO timer will never expire prematurely. We then have only retransmission due to loss. From Equation 4, the throughput is maximized when $\tau_{R T O}$ is minimized, so $\tau_{R T O}$ should be set to $m$. For $p_{l}=0$, we have the case of spurious retransmission only. Then $T\left(\tau_{R T O}\right)$ is a monotonically increasing function, and the throughput approaches the maximum value of one for $\tau_{R T O} \rightarrow \infty$. When both types of retransmission are present, the optimal setting for $\tau_{R T O}$ will be somewhere between 1 and $x$.

From Equation 3, it is easy to see that the throughput depends on $\sigma_{d}^{2}$ and on the tail of $P(t)$. To illustrate the effect of these two attributes, we consider three representative probability density functions, for the case $m=5$.

\subsection{Gaussian Delay Distribution}

The Gaussian distribution has been used to model the delay experienced by packets traversing multiple switches where there are queueing delays. When the number of the switches en route is large, the total delay can be approximated by the Gaussian distribution. This distribution falls off very rapidly, as $e^{-t^{2}}$. If the packet goes through $N$ switching nodes, each with identical delay distribution, then both the end-to-end delay mean and variance will grow linearly with $N$, so that the normalized standard deviation will decrease as $\frac{1}{\sqrt{N}}$. For the case of Gaussian delays. we have

$$
\begin{aligned}
P(t) & =\sqrt{2} \sigma \int_{\infty}^{t} e^{\frac{(x-m)^{2}}{2 \sigma^{2}}} d x \\
& =\frac{1}{2}+\sqrt{2} \sigma \operatorname{erf}\left(\frac{t-m}{\sqrt{2} \sigma}\right) .
\end{aligned}
$$

\footnotetext{
${ }^{3}$ We are ignoring losses due to buffer overflow.
}

where erf $(t)$ is the error function[10]. Then the probability of spurious retransmission is

$$
p_{t}=\sqrt{2} \sigma \operatorname{erfc}\left(\frac{\tau_{\text {KTO }}-m-1}{\sqrt{2} \sigma}\right)
$$

Figure 1 shows the throughput as a function of $\tau_{R T O}$ for $\sigma_{d}^{2}=\frac{1}{4}$ and a range of $p_{l}$.

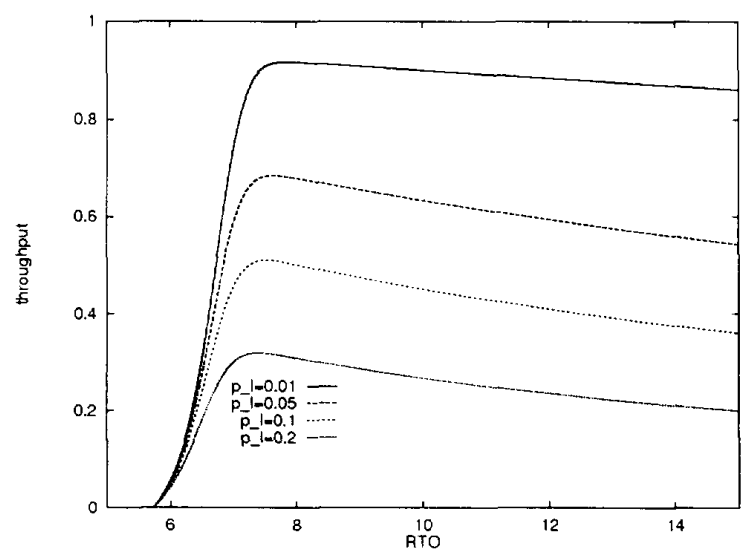

Figure 1: Throughput for gaussian delays, $m=5$ and $\sigma_{d}=\frac{1}{2}$.

\subsection{Exponential Delay Distribution}

The exponential distribution falls off slower than the Gaussian one, as $e^{-t}$. An $M / M / 1$ queue has such a distribution for the delay, so it is a good model for the case the packet only goes through one switching node. For the case of exponential delays, we have

$$
P(t)=1-\epsilon^{-\frac{t}{m}}
$$

and the probability of spurious retransmission is

$$
p_{d}=\epsilon^{-\frac{\tau_{R T O-1}}{m}} .
$$

Figure 2 shows the dependence of the throughput on $\tau_{R T T}$ and on $p_{l}$, the packet loss probability, for the exponential delay distribution.

\subsection{Hyperexponential Delay Distribution}

The hyperexponential distribution has even higher variability in delay, and is a good model for the case of link level retransmission, which will result in the end-to-end delay having a large variance. For the case of hyperexponential delays. we have

$$
P(t)=1-\alpha_{1} \epsilon^{-\mu_{1} t}-\alpha_{2} \epsilon^{-\mu_{2} t}
$$

with $\alpha_{1}+\alpha_{2}=1$ and $\frac{\alpha_{1}}{\mu_{1}}+\frac{\alpha_{2}}{\mu_{2}}=m$. The probability of spurious retransmission is

$$
p_{d}=\alpha_{1} f^{-\mu_{1}\left(\tau_{R T O}-1\right)}+\alpha_{2} \epsilon^{-\mu_{2}\left(\tau_{R J O}-1\right)} .
$$




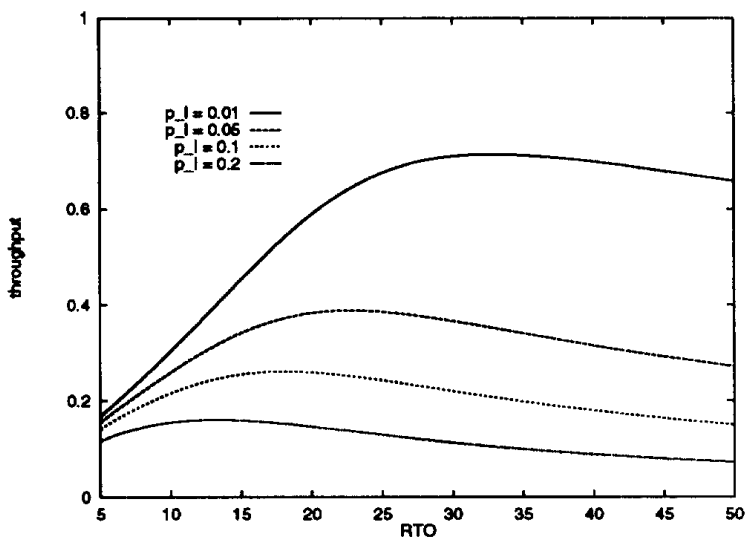

Figure 2: Throughput for exponential delay, $m=5$.

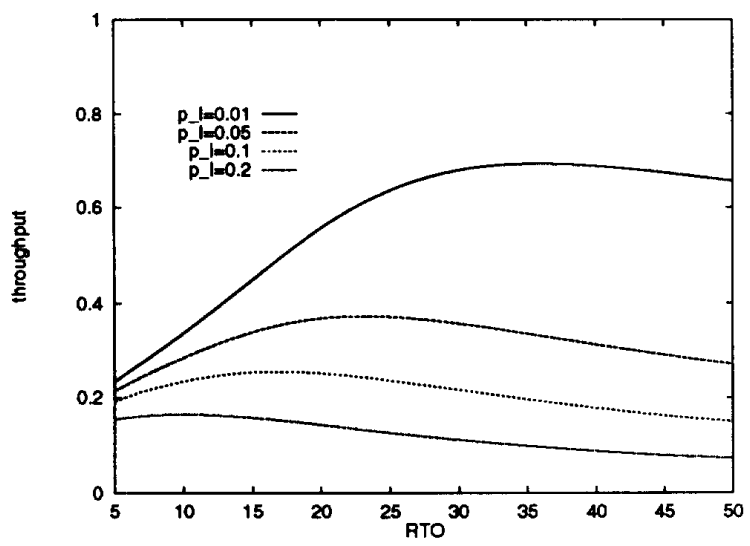

Figure 3: Throughput for hyperexponential delay with $m=5$ and $\sigma_{d} \approx 5.6$.

Figure 3 shows the throughput as a function of $\tau_{R T O}$ for $p_{l}=0.1$, and $\alpha_{1}=0.9, \mu_{1}=1.3$ and $\mu_{2}=0.325$, giving a $\sigma_{d}^{2}$ of about 2 .

Figures 1, 2 and 3 show that, for all the delay distributions, as $p_{l}$ increases, the optimal value of $\tau_{R T O}$ decreases, as timely recovery from loss begins to dominate over spurious retransmission. For the same loss rate and mean delay, increased variability in delay decreases the throughput of the transport protocol at the optimal value of $\tau_{R T O}$. Further, for a given mean delay the optimal value of $\tau_{R T O}$ shifts to larger values as the delay variability increases.

\section{TCP Performance with Link-Layer Re- transmissions}

To investigate the behavior of a transport protocol in the presence of link-layer retransmissions, we consider a simple transport connection over two links (Figure 4).
Packets may be lost on either link. We interpret link $a$ as a wireless link: it has an inherently higher error rate and lower data rate than link $b$, which we interpret as a wired link. The transmitting host uses timer-based retransmissions for error recovery, calculating the $\tau_{R T T}$ and $\tau_{R T O}$ according to some algorithm. For simplicity we ignore congestion: all losses are due to errors and not congestion. We assume that the transmitter uses sliding window flow control.

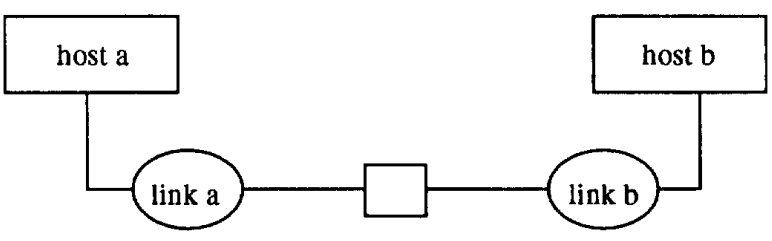

Figure 4: An end-to-end connection

We denote the round-trip packet transmission delay on links $a$ and $b$ as $\tau_{a}$ and $\tau_{b}$, respectively. Similarly, $p_{a}$ and $p_{b}$ are the corresponding packet loss rates, with $p_{a} \gg p_{b}$, and $C_{a}$ and $C_{b}$ are the link speeds in packets per second. The round-trip delay for the case without LLR is

$$
\tau_{R T T}=\tau \equiv \tau_{a}+\tau_{b}
$$

With LLR, the round-trip delay is

$$
\tau_{R T T}=\eta \tau_{a}+\tau_{b}
$$

where $\eta$ the number of times a packet was transmitted. For random packet losses, $\eta$ has the geometric distribution[11]:

$$
\operatorname{Pr}(\eta=n)=p_{a}^{n-1}\left(1-p_{a}\right), \quad n=1,2, \ldots
$$

We look at the effects of retransmission on TCP's calculation of $\tau_{\text {RTO }}$ in Section 5.2.

\subsection{TCP}

The importance of the $\tau_{R T T}$ and $\tau_{R T O}$ are recognized for TCP[12], and clever methods have arisen to estimate the $\tau_{R T T}$ and to calculate the $\tau_{R T O}$ based on $\tau_{R T T}$ estimates[13][14]. The TCP specification[4] proposed an exponentially weighed-moving average for the $\tau_{R T T}$. Each end-to-end acknowledgment produces a measurement of the round-trip time, $\tau_{i}$, and the estimate of $\tau_{R T T}$ used in calculating $\tau_{R T O}$ is:

$$
\tau_{R T T}^{(i+1)}=\alpha \times \tau_{i}+(1-\alpha) \times \tau_{R T T}^{(i)} .
$$

The TCP spec[4] suggests a gain, $\alpha$, of 0.1 and calculation of the $\tau_{\text {RTO }}$ as $\beta \times R$ with $\beta \approx 2$.

Jacobson[13] augments the $\tau_{R T T}$ calculation with an estimate of the mean deviation in the $\tau_{R T T}$ :

$$
\delta_{R T T}{ }^{(i+1)}=\alpha_{\delta} \times\left|\tau_{i}-\tau_{R T T}{ }^{(i)}\right|+\left(1-\alpha_{\delta}\right) \times \delta_{R T T}{ }^{(i)},
$$


and suggests $\alpha_{\delta}=\frac{1}{4}$ for the gain in the mean deviation calculation. ${ }^{4}$ Jacobson's paper calculates the retransmission timeout as ${ }^{5}$

$$
\tau_{R T O}{ }^{(i)}=\tau_{R T T}{ }^{(i)}+2 \times \delta_{R T T}^{(i)} .
$$

The Host Requirements RFC[12] suggests initial values of $\tau_{R T T}{ }^{(0)}=1 \mathrm{sec}$ and $\delta_{R T T}{ }^{(0)}=1 \mathrm{sec}$, so $\tau_{R T O}{ }^{(0)}$ is 3 seconds. In addition, TCP implementations must also include "exponential backoff" for successive $\tau_{R T O}$ values for the same segment[12].

Karn's algorithm[14] modifies Equation 16 to eliminate the effects of "ambiguous" $\tau_{R T T}$ measurements due to retransmitted packets. A measurement for a retransmitted packet is ambiguous since the acknowledgment carries no information on which transmitted packet is being acknowledged. A conservative assumption is to measure $\tau_{i}$ from the first transmission, but this inflates the $\tau_{R T O}$ which reduces performance. Simply ignoring estimates from retransmitted packets is not an adequate solution since the $\tau_{R T T}$ and $\tau_{R T O}$ estimates will grow worse at the worst possible time: when congestion in the network is causing packet losses. Karn's algorithm avoids this difficulty by, in addition to ignoring $\tau_{R T T}$ measurements from retransmitted packets, using the value of the $\tau_{r T O}$ for the retransmitted packet (after backoff) as the $\tau_{R T O}$ for the next packet to be transmitted. The "backed off" $\tau_{\text {RTO }}$ will continue to be used until a acknowledgment is received for a packet transmitted exactly once. Then the $\tau_{R T T}$ is updated with the new, unambiguous sample and the $\tau_{R \text { RO }}$ from Equation 16 is used for subsequent packets.

\subsection{Behavior of $\tau_{R T T}$ and $\tau_{R T O}$ estimates}

Before we show the throughput results for dynamic $\tau_{R T O}$ from our simulation, we investigate the behavior of $\tau_{R T T}$ and $\tau_{R T O}$ in the presence of LLR.

Equations 14 and 15 can be rearranged, using Equation 12 , to give:

$$
\begin{aligned}
& \tau_{R T T}{ }^{(i+1)}-\tau_{R T T}{ }^{(i)}=\alpha\left(r_{a} \eta_{i}+r_{b}-R_{i}\right) \\
& \delta_{R T T}{ }^{(i+1)}-\delta_{R T T}{ }^{(i)}=\alpha_{\delta}\left(\left|\eta_{i} \tau_{a}+\tau_{b}-\tau_{R T T^{(i)}}\right|-\delta_{R T T}{ }^{(i)}\right)
\end{aligned}
$$

Note that in Equation 17, $\tau_{a}$ only enters in the combination $\eta_{i} \tau_{a}$; that is, it only scales the contribution of the stochastic term. It does not change the equilibrium dynamics of the system. We are not investigating the

\footnotetext{
${ }^{4}$ Jacobson[13] also suggests that using a reciprocal power of 2 for $\alpha$ as well would make implementation more efficient, and proposes $\frac{1}{8}$. We will use $\alpha=\frac{1}{8}$ and $\alpha_{\delta}=\frac{1}{4}$ in what follows.

${ }^{5}$ This is an improvement to the method for calculating the $\tau_{R T O}$ suggested in the TCP spec [4], where $\tau_{R T O}$ is set to a small multiple of the $\tau_{R T T}$.
}

dependencies on $\alpha$ and $\alpha_{\delta}$, and use the standard values quoted in Section 5.1: $\alpha=\frac{1}{8}$ and $\alpha_{\delta}=\frac{1}{4}$. The only interesting parameter left is the packet loss probability, $p$.

To investigate the behavior of Equation 17, we generate samples of the sequence $\left\{\eta_{i}\right\}$, and use Equation 17 to generate the sequences $\left\{R_{i}\right\}$ and $\left\{\delta_{i}\right\}$. The sequences $\left\{R_{i}\right\}$ and $\left\{\delta_{i}\right\}$ in turn determine the $\tau_{R T O}$

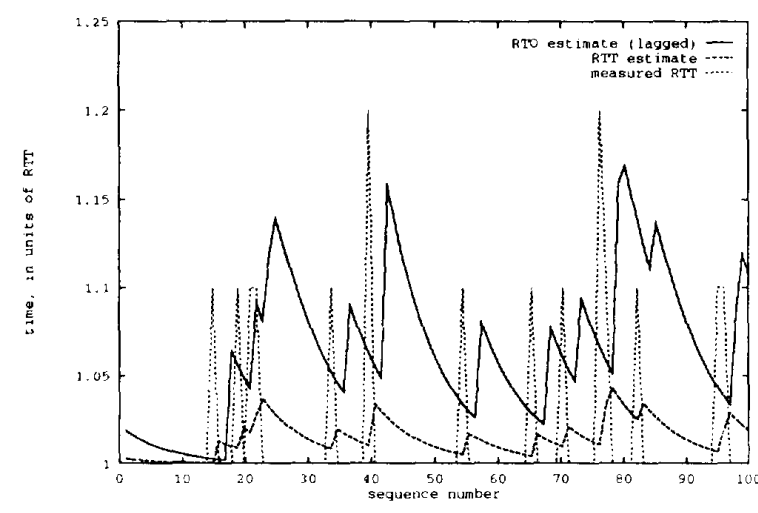

Figure 5: Behavior of $\tau_{R T O}: p=0.1$

The $\tau_{\text {RTO }}$ also depends on two other factors[14]:

- The scheme used to accommodate retransmitted packets in the $\tau_{R T T}$ calculations.

- The strategy for adjusting the $\tau_{R T O}$ for packets transmitted after expiry.

The simplest approach is to measure $\tau_{R T T}$ from the first transmission, and leave the $\tau_{R T O}$ for subsequent packets unmodified after detecting loss. This has well-known problems[14]. Karn's algorithm, discussed in Section 5.1, addresses these problems, but complicates the interpretation of the $\tau_{\text {RTO }}$.

According to the Host Requirements[12], on expiration of the timer, the packet's $\tau_{R T O}$ is backed off. In this section we ignore the possibility of multiple TLRs for the same packet. We use binary exponential backoff when implementing the Karn correction to assign a $\tau_{R T O}$ to packets transmitted after an expiry.

Determining the end-to-end performance requires calculation of the $\tau_{R T O}$, which again is a random sequence $\left\{\tau_{R \text { To }}{ }^{(i)}\right\}$, calculated according to Equation 16. The $i^{\text {th }}$ packet will be retransmitted if the packet's $\tau_{R T T}$ is greater than the value of the $\tau_{R T O}$ at the time the packet was sent. We count a spurious retransmission whenever

$$
\tau_{i}>\tau_{R T O}{ }^{(i-w)}
$$

where $w$ is the window size, in packets. 
Figure 5 shows a typical sample path for the roundtrip times, according to Equation 12, along with the estimate of $\tau_{R T O}$ calculated using estimates of $\tau_{R T T}$ measured from the first transmission (without the Karn correction). Whenever the sample exceeds $\tau_{R T O}$, a spurious end-to-end retransmission is generated for a packet that was retransmitted on the noisy link.

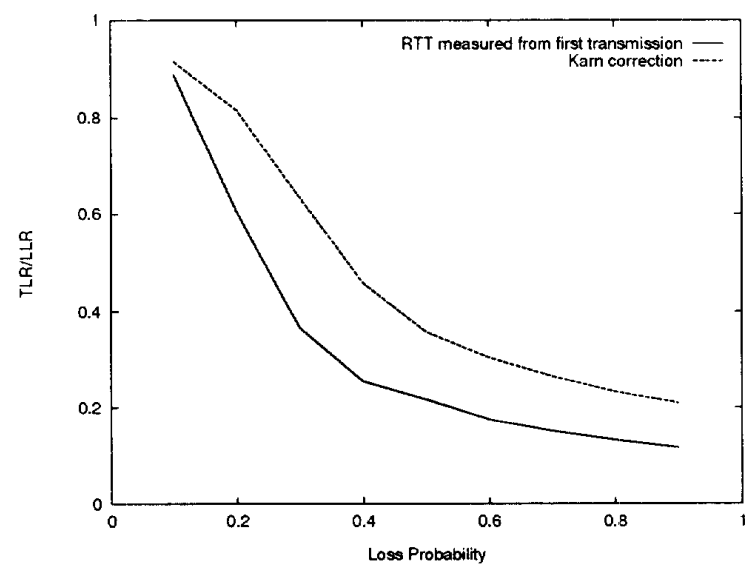

Figure 6: Ratio of transport-level retransmissions to link-level retransmissions.

As a measure of the effect of LLR on the usable capacity of the noisy link, consider Figure 6, which shows the fraction of packets retransmitted on the link that are also retransmitted end-to-end. The solid line is for roundtrip times calculated from the first transmission, and the dashed line includes the Karn correction, discussed in the text. At low loss rates, almost all packets retransmitted on the link are retransmitted end-to-end as well. The excess traffic generated by the redundant retransmissions represents wasted capacity on the link.

\subsection{Simulation Results}

We developed simulation models using $\mathrm{Q}^{+}$, a simulation tool developed at AT\&T Bell Laboratories[15]. Using simulation, we can model a more realistic transport protocol, and we can evaluate the system performance under a variety of conditions: round-trip time variability, different link capacities on the wired and wireless links, cross traffic effects, etc.. We are also interested in the role of different transport-layer mechanisms, such as dynamic window sizes and slow start, that are part of the TCP approach to congestion control. The simulation experiments help us to identify the packet loss rate range where the LLR is useful to a single TCP connection and evaluate the cost of using the LLR in terms of additional load on the wireless link.

Figure 7 shows a $\mathrm{Q}^{+}$model used in the simulation study of the two-link end-to-end path described above.

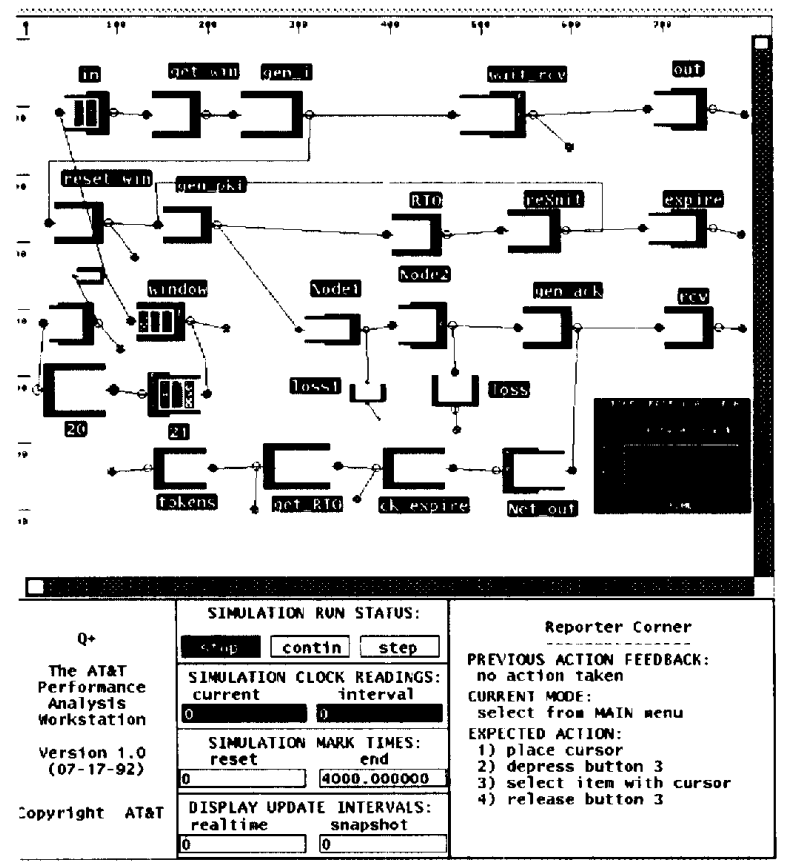

Figure $7:$ Q+ simulation model for TCP

The transmitter uses timer-based retransmissions for endto-end error recovery, calculating RTT and RTO according to Equations 14-16. We use the Karn correction to calculate $\tau_{\text {RTO }}$ when a timeout occurs. For the link layer retransmissions, we assume that the transmitter at the wireless link uses a stop-and-wait protocol, where negative acknowledgment is used as an indication of a packet loss.

We assume further that the transmitter host always has packets ready to transmit. We also assume that all losses are due to independent errors, and errors only occur on the wireless link. The performance measures that we are interested in are: (i) the application throughput, defined as the rate at which the transport protocol delivers packets to the application layer, (ii) the link cost, defined as the ratio of the number of packets transmitted on the wireless link to the number of packets received by the application.

As a simple case, consider a transport protocol with fixed window size and adaptive timeout calculation. We use a window size of 5 packets. Figure 8 shows the application throughput as a function of packet error rate for deterministic link delays and a slow wireless link. For packet loss rates of less than $12 \%$, the throughput of a system with TLR only is higher than the throughput of a system with both TLR and LLR. For the same mean link delays but an exponential delay distribution on the wire- 


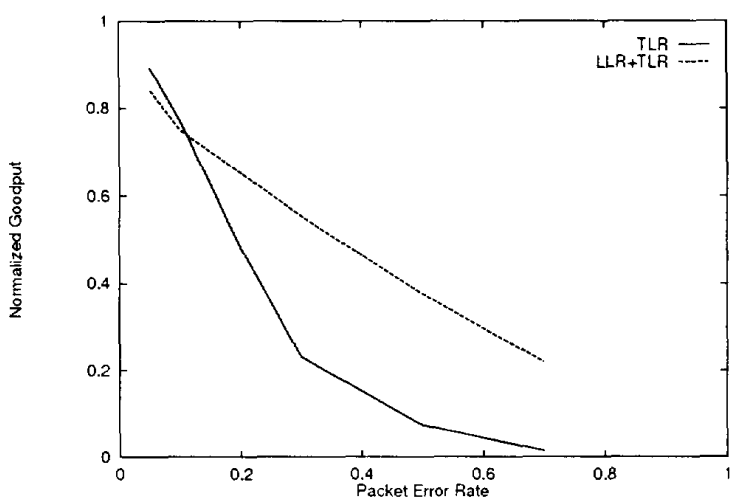

Figure 8: Throughput for fixed windows, deterministic link delays and a slow wireless link $\left(\tau_{a}=0.7\right.$, $\tau_{b}=0.3$ )

less link, the throughput of TLR only is higher for packet error rates of less than $10 \%$. Figure 9 shows the plot for the cost of of having LLR (i.e. the plot of the ratio of total wireless link transmissions to total received), again for deterministic link delays and a slow wireless link. The figure shows that, at $10 \%$ packet loss, while the end-toend throughput is about the same, each successful packet reception requires about 1.12 link transmissions when using TLR only, and 1.31 when using both LLR and TLR.

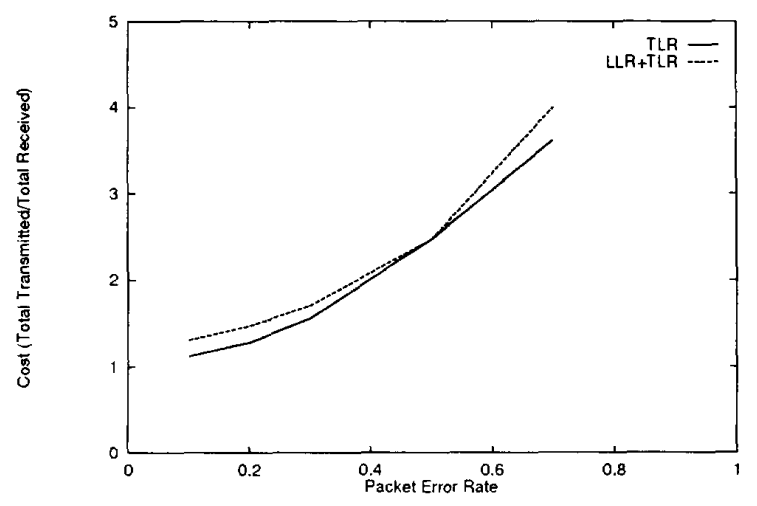

Figure 9: Number of link transmissions per successful end-to-end transmissions

Next, we compare the results we get with fixed window with another version of the model whereby we use a $d y$ namic window mechanism in the end/end protocol, nodeled after TCP. The dynamic window mechanism starts from a window size of one and increments the window

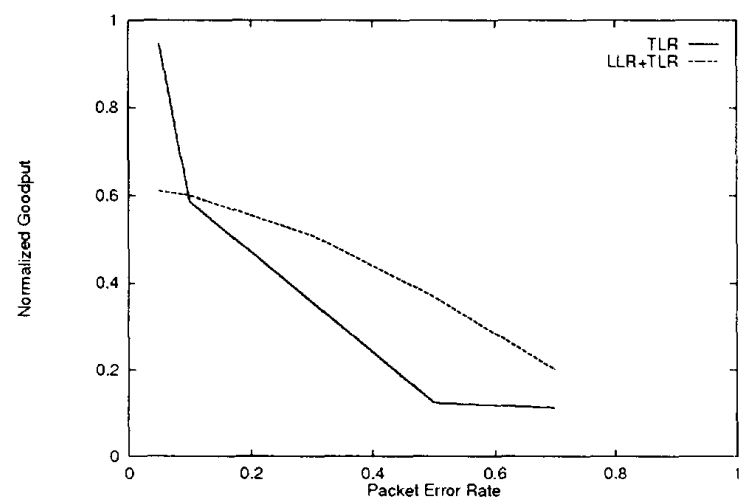

Figure 10: Throughput for dynamic windows, deterministic link delays and a slow wireless link $\left(\tau_{a}=\right.$ $\left.0.7, \tau_{b}=0.3\right)$

size upon receiving a positive acknowledgment, up to the maximum window size (chosen to be 5 , just as in the fixed window experiments). The window size shrinks to one upon the expiration of the timer, similar to TCP's slow-start behavior.

Figure 10 shows the throughput results with dynamic windows, for the same system parameters as Figure 8 . The comparison of TLR alone to TLR with LLR is not very different than in Figure 8 , but dynamic windows further reduce the throughput due to losses and timeouts.

\section{Conclusions}

Link layer retransmission has been proposed as one way to improve the error rate performance of the radio link in a wide area network. This paper points out some of the complexities in the interaction of multiple levels of protocols. We have intentionally focused on TCP as an example of a transport protocol because of its wide deployment over a variety of networks and its use to support a wide variety of applications, from simple terminal sessions to graphical terminal to electronic mail and file transfer. We expect the performance in supporting existing TC:P hosts an important measure of any new networking technologies, particularly the emerging wireless networks.

Our results point out some of the complicated interactions between end-to-end transport protocols and linklevel protocols. We show how, for fixed $\tau_{R T O}$, increased variability in delay decreases the throughput of the transport protocol at the optimal value of $\tau_{R T O}$. For adaptive $\tau_{R T O}$ in the manner of TCP, we show how spurious TLRs reduce the usable capacity of a link that does LLR. 
In our study, we are interested in two metrics, the throughput seen by the applications (defined to be the rate at which packets can be transmitted by the TLR protocol) and the cost (defined to be the number of packets that need to be transmitted at the wireless link to the number of packets that actually get transmitted by the TLR protocol). Our results indicate that the application throughput improves with the presence of the LLR protocol only when the packet error rate exceeds a certain threshold. This threshold is dependent on various factors e.g. whether we have fixed/dynamic windows for TLR, whether we have slow start/no slow start for TLR. The next question to ask ourselves is the cost of achieving this improvement in the throughput. At small error rate, almost all the packets that are retransmitted by LLR are retransmitted by TLR, but the ratio of TLR/LLR retransmitted packets drop very fast with increasing error rate.

In our analysis and simulation we have assumed the packet losses to be statistically independent. Since the loss mechanisms are fading in the radio environment and buffer overflows in the network, we shall study the throughput for cases of correlated packet losses. Further research in the area of transport layer performance over wireless networks can also include new algorithms for adjusting $\tau_{R T O}$ dynamically.

\section{References}

[1] D. J. Goodman, "Trends in cellular and cordless comunications", IEEE Communications Magazine. pp. 31-40, June 1991.

[2] D. C. Cox, "Universal portable radio communications", Proceedings of the IEEE, pp. 436-477, April 1987.

[3] S.H. Jamali and T. Le-Ngoc, "Performance comparison of different decoding strategies for a bandwidthefficient block-coded scheme on mobile radio channels", IEEE Tran. on Vehicular Technology, Vol. 41, No. 4 pp 505-515, Nov 1992.

[4] J. B. Postel, "Transmission control protocol", RFC 793, SRI Network Information Center, September 1981.

[5] Stephan William Edge, "Comparison of hop-by-hop and endpoint approaches to network interconnection", in J.-L. Grangé and M. Gien, editors, Flow Control in Computer Networks, pp. 359-377. NorthHolland, 1979.

[6] Subir K. Biswas, John D. Porter, and Andy Hopper, "Performance of a multiple access protocol for an atm based pico-cellular radio lan", The Third IEEE International Symposium on Personal, Indoor and Mobile Radio Communications, Oct 1992.
[7] J.J. Bae, T. Suda, and N. Watanabe, "Evaluation of the effects of protocol processing overhead in error recovery schemes for a high-speed packet switched network: Link by link versus edge to edge schemes", IEEE J. Selected Areas in Communications, Vol. 9, No. 9 pp 1496-1509, Dec 1991.

[8] Bharat T. Doshi, Pravin Johri, Arun N. Netravali, and Krishan K. Sabnani, "Error and flow control performance of a high speed protocol", IEEE Transactions on Communications, vol. 41, pp. 707-720, May 1993.

[9] Mischa Schwartz, Telecommunications Networks: Protocols, Modeling and Analysis, Addison-Wesley Series in Electrical and Computer Engineering. Addison-Wesley, 1987.

[10] Milton Abramowitz and Irene A. Stegun, Handbook of mathematical functions with formulas, graphs, and mathematical tables, Number 55 in Applied Mathematics Series. National Bureau of Standards, June 1964.

[11] Sheldon M. Ross, Introduction to Probability Models, Academic Press, fourth edition, 1989.

[12] R. Braden, "Requirements for internet hosts - communication layers", RFC 1122 , SRI Network Information Center, October 1989.

[13] Van Jacobson, "Congestion avoidance and control", in Communications, architectures E protocols, pp. 314-329. ACM SIGCOMM, August 1988.

[14] Phil Karn and Craig Partridge, "Improving roundtrip time estimates in reliable transport protocols", ACM Transactions on Computer Systems, vol. 9, pp. 364-373, November 1991.

[15] Cynthia A. Funka-Lea, Tasos D. Kontogiorgos, Robert J. T. Morris, and Larry D. Rubin, "Interactive visual modeling for performance", IEEE Software, pp. 58-68, September 1991. 